\title{
Biomechanical Indicators of Competitive Activity in Mas-Wrestling
}

\author{
Loginov V.N. \\ Department of Sports Training and National Sports \\ Churapcha State Institute of Physical Culture and Sport \\ Churapcha, Russia \\ lognic@rambler.ru
}

Fedorov E.P.

Department of Natural Sciences

Churapcha State Institute of Physical Culture and Sport

Churapcha, Russia

ffep@mail.ru

\author{
Pyanzin A.I. \\ Department of Theoretical Basis of Physical Education \\ Chuvash State Pedagogical University n.a. I.Y. Yakovlev \\ Cheboksary, Russia \\ pianzin@mail.ru
}

\author{
Atlaskin A.L. \\ Department of Physical Culture and Sport \\ Chuvash State University n.a. I.N. Ulyanov \\ Cheboksary, Russia
}

\begin{abstract}
The paper assesses the possibilities of using biomechanical indicators of competitive activity in maswrestling (the relative position of stick fluctuation zones and the general center of mass (GCM) of two athletes' bodies system, the angle changes in the knee and hip joints) to determine the technical and tactical advantages (tactical efficiency, coordination of movements and economy of technical actions) of athletes. The discrepancy between the stick position zones and the general center of mass of the twobody system is revealed. The most significant is the ratio indicator of positions number of the general center of mass of bodies system to the number of stick positions on a certain side, reflecting the tactical efficiency of an athlete. In the winner of the fight, it was 5.5 times higher than in the loser. Tactical efficiency, in turn, is determined by the coordination of athlete's body links movements in the knee and hip joints. In the winner, the bending of the torso is synchronized with the bending of the legs and vice versa. The loser does not have such consistency, he either extends his legs, not reinforcing it by extension of the body, or extends the body without the help of the legs. Economy of technical actions reflects the modulus of difference fluctuations in the angles in these joints. The average value of this indicator for the loser is $15.2 \pm 11.98^{\circ}$, for the winner $-8.4 \pm 6.65^{\circ}$, almost 2 times lower $(p<0.05)$.
\end{abstract}

Keywords-mas-wrestling; competitive activity; biomechanical indicators; tactical efficiency; coordination of movements; economy of technical actions.

\section{INTRODUCTION}

One of the central problems in mas-wrestling is the search for ways to improve technical and tactical skills. Mas-wrestling is stick pulling by opponents in two or three support positions. The external simplicity of movements in mas-wrestling hides the technically difficult wrestling and maximum muscle tension.

As in all types of wrestling, two athletes take part in mas-wrestling. The peculiarity of the competitive battle in this type of wrestling is that the athletes are in constant contact from its beginning to the end, that is, they make up a relatively balanced system of two bodies, pushing their legs away from the support board and pulling the stick to one's side with hands.

The task of each of the opponents is to unbalance this system by any means permitted by the competition rules, tearing the stick out of the opponent's hands, or dragging it to one's side. Rupture of this ligament means the end of the scrum. The struggle itself looks dynamic and is characterized by a rapid change of scenery.

The scientific and methodological literature analysis showed that studies in mas-wrestling are mainly focused on finding ways to improve the technique of motor actions during the struggle $[1,2,3,4,5,6,7,8,9]$. At the same time, in our opinion, the process of the struggle itself has not been sufficiently studied from the biomechanical point of view, which makes it difficult to find new ways to improve the technical and tactical skills of athletes. 


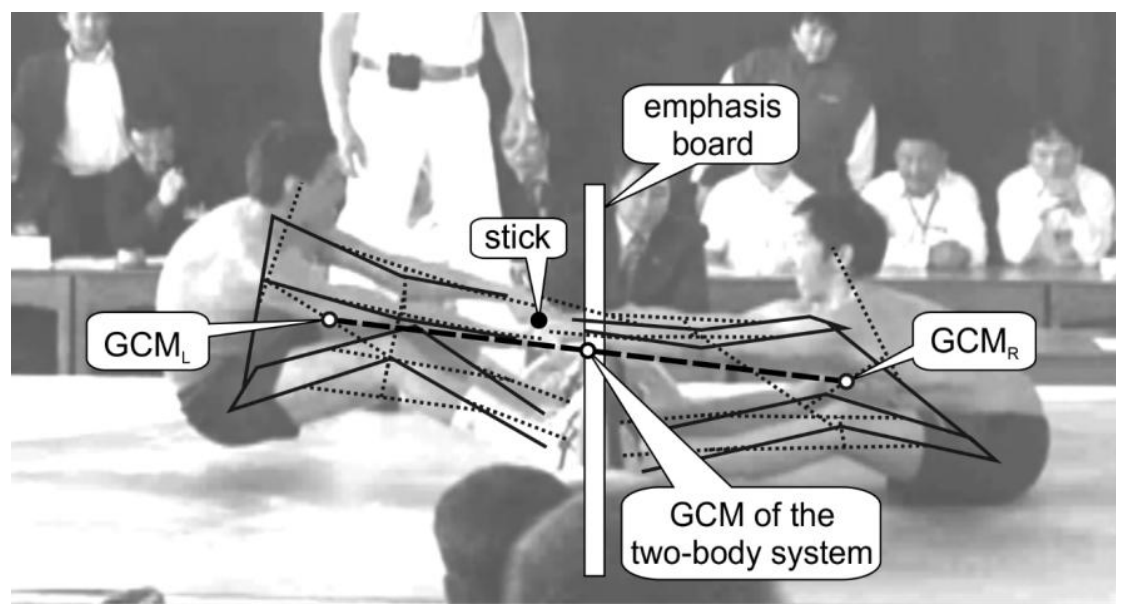

Fig. 1. Location of support board, stick, gcm of two-body system and $\mathrm{gcm}$ of each of the opponents in the $3^{\text {rd }}$ second of fight

In this regard, the aim of the study was to assess the possibility of using biomechanical indicators of competitive activity in mas-wrestling to determine the technical and tactical advantage.

Object of research - competitive activity in maswrestling.

Subject of research - biomechanical indicators of competitive activity in mas-wrestling.

Research objectives:

1. To identify the tactical effectiveness of the rivals actions based on the comparison of the stick trajectories and the general center of mass of athletes' two-bodies system.

2. To evaluate the consistency of body parts movements and the economy of athlete's technical actions based on the comparison of angle changes in the knee $(\mathrm{KJ})$ and hip joints $(\mathrm{HJ})$.

\section{RESEARCH METHODOLOGY}

To achieve the goal and solve the tasks we used a video of one of the competitive fights, shot with a fixed camera, which was located on the side of athletes along the axis of the support board. Separate frames with a time interval of 1.00 seconds were selected from the video. The total duration of the scrum was 21 seconds.

The frames were processed in the CorelDRAW graphical editor using linear graphics tools that made it possible to superimpose the position of the support board, sticks, 14-link diagram of the human body, as well as to determine the location centers of mass of individual body parts and to designate the location of each athlete's body GCM and the GCM of two-body system (Fig. 1).

The presence in the graphics editor of convenient and accurate tools for measuring linear and angular quantities made it possible to determine the coordinates of particular points and their trajectories.
Due to the lack of a clearly defined amplitudinous scale in the location area of athletes, all linear dimensions were measured on the image in millimeters without determining its scale for converting distances to real values. In our study, the need to measure real distances was not principled.

\section{RESULTS}

Video analysis allowed us to note that the athlete on the left uses an aggressive attack tactical fight at a torn pace, holding the stick on his side with a sufficiently large deviation from the initial average position, while the athlete on the right uses defensive tactics, giving the opponent the position of the stick, but not giving him the final advantages needed to win the scrum. As a result, it was the right athlete who won that scrum, despite the lack of visual advantage during the fight. What technical and tactical actions allowed this athlete to win?

As part of solution of the $1^{\text {st }}$ research task, we analyzed the trajectory of the stick and the GCM of two athletes bodies system. Figure 2 shows the trajectories of the stick and the GCM of the system relative to the initial position corresponding to zero coordinates. Already an overall look at this figure allows us to note the discrepancy between the zones of stick positions and the GCM of two-body system. The stick positions zone is located to the left of positions zone of the GCM of system located in the region of the zero coordinate.

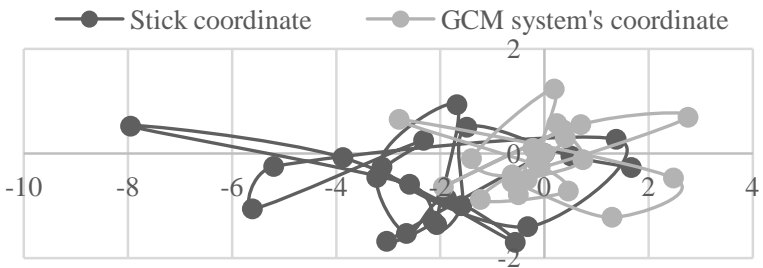

Fig.2. Trajectories of movement of the stick and the GCM of two-body system during the competitive bout 


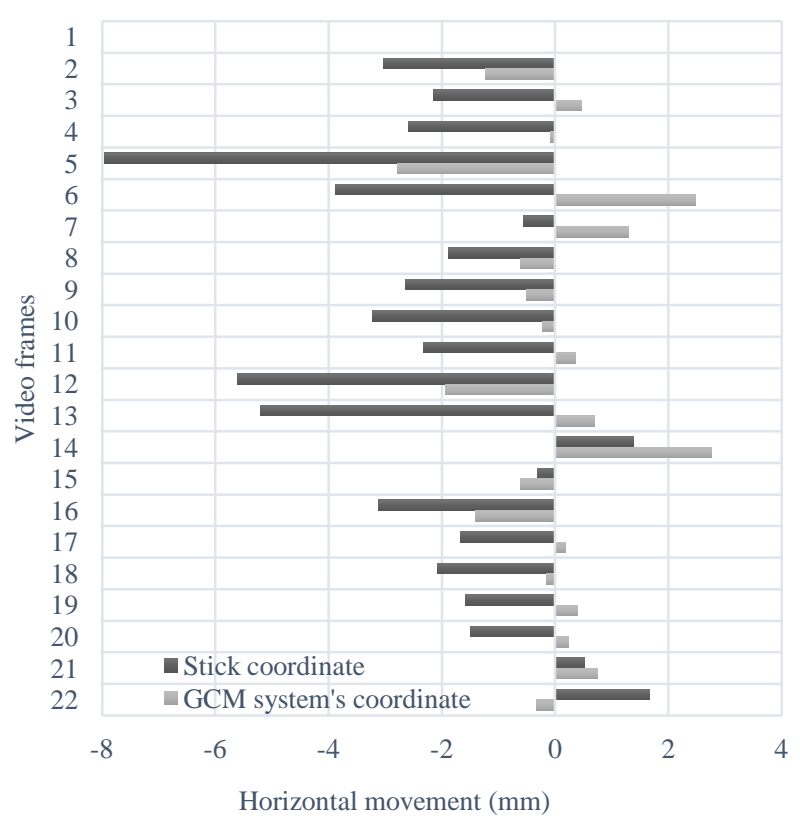

Fig.3. Horizontal movements of a stick and the GCM of two-body system during the competitive bout

The number of stick positions and the GCM of two-body system was calculated on each side of the zero coordinate in the course of the scrum (Fig. 3). At the first blush, the athlete on the left has a clear advantage in the number of stick positions on his side (18 to 3, i.e. 6 times more often than the opponent on the right), and relative parity in the number of positions of the GCM of the two-body system (11 to 10$)$.

But, taking into account that the right athlete won in the end, we can assume that the position of the GCM of the system, and not of the stick, is a more significant indicator. Even more significant is the ratio indicator of positions number of the GCM of bodies system to the number of positions of the stick on a certain side. If the stick is more often on the left of the starting position at the beginning of the scrum, and the GCM of bodies system is on the right, then the athlete on the right is more tactically engaged in tactical fight, allowing the athlete on the left to have a visual advantage in the stick position, but not giving him the advantage of fighting in the position of the two-body system GCM.

By dividing the positions number of the GCM of bodies system by the number of stick positions from one of the opponents, we can calculate his tactical efficiency. The tactical efficiency of the athlete on the left is equal to the ratio of the number of left-side positions of the GCM of two-body system (11) to the number of left-side stick positions (18) during the scrum. It is equal to 0.61 units. The tactical efficiency of the athlete on the right is equal to the ratio of the number of right-handed positions of the GCM of two-body system (10) to the number of right-handed stick positions (3) during the fight. It is 3.33 units. According to this indicator, the athlete on the right has a significant advantage. His tactical efficiency is 5.5 times higher.
The question arises, due to what the athlete on the right received such a significant advantage? We assume that his tactical advantage reflects a higher level of technical actions execution.

To confirm the assumption, there was made an assessment of body parts movements consistency of each of the athletes based on the comparison of angle changes in the knee and hip joints ( $2^{\text {nd }}$ study objective).

The flexion-extension of the legs and torso of the athlete on the right occurs relatively synchronously, as indicated by the presence of positive and negative peaks at the same time points. That is, the bending of the body occurs simultaneously with the bending of the legs and vice versa. The athlete on the left has no such consistency in changing the angles in the joints. He either extends his legs, not reinforcing it with an extension of the torso, or extends his torso without the help of his legs. Perhaps the athlete on the left lacked a more coordinated work of body parts to bring the fight to victory.

The consistency of body link movements was evaluated using the nonparametric Z-criterion of signs. Coordinated movement in this context is manifested in the simultaneous flexion (extension) in both joints, non-coordinated movement is manifested in extension in one joint when bending in the other. As a result, the coordination of movements turned out to be reliable for the athlete on the right $(\mathrm{p}<0.01)$ and not reliable for the athlete on the left $(\mathrm{p}$ $>0.05)$.

The angle changes in the joints made it possible to evaluate such an indicator as technique economy. For this, a difference between angles in the knee and hip joints at each moment of the scrum was revealed. Given a less coordinated work of the legs and torso of the athlete on the left, difference fluctuations in the angles of $\mathrm{KJ}-\mathrm{HJ}$ are more significant than those of his opponent. But for us, it was not so much the difference in angles that was important as the changes in this difference relative to the previous moment of the scrum, taken modulo (Fig. 4).

The average values of this indicator for the athlete on the left are $15.2 \pm 11.98^{\circ}$, for the athlete on the right $-8.4 \pm$ $6.65^{\circ}$, almost 2 times lower $(\mathrm{p}<0.05)$, which may indicate a more economical fight conducting manner of the athlete on the right, who forces the athlete on the left to spend more effort, exhausting him and saving more of his own strength for the final moments of the fight. 


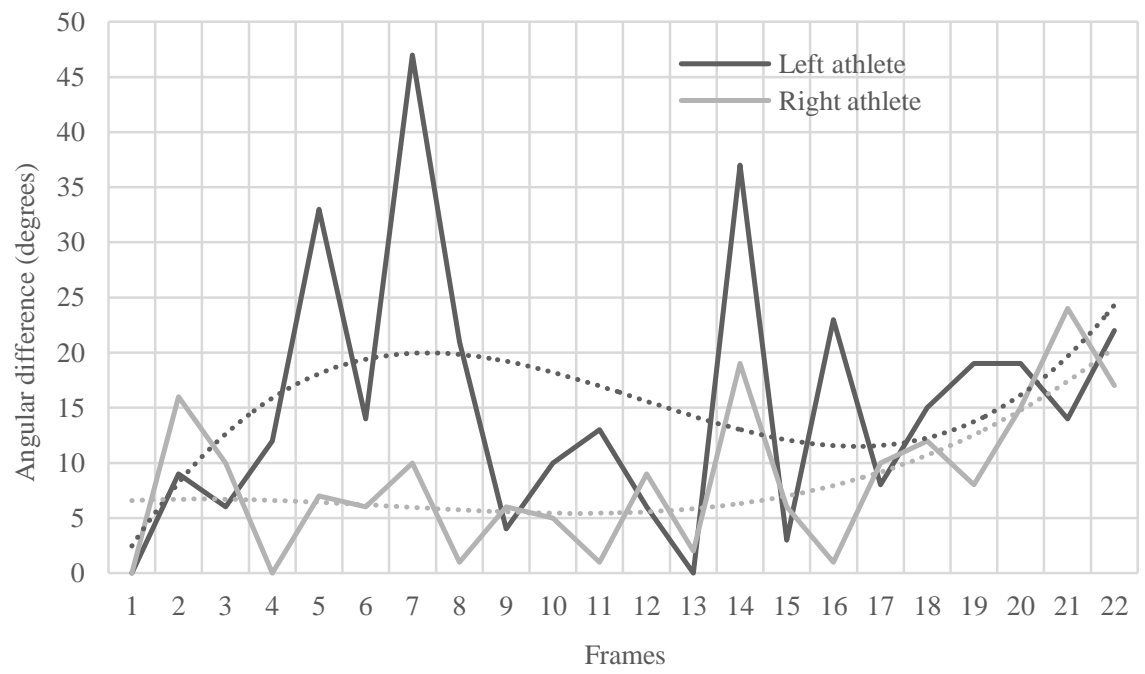

Fig.4. Module of angles difference fluctuations in the knee and hip joints of opponents during the bout

\section{CONCLUSION}

Thus, to determine the technical and tactical advantage (tactical efficiency, movements coordination and the economy of technical actions) in mas-wrestling, biomechanical indicators of competitive activity can be informative: the relative position of the stick fluctuation zones and the general center of two athletes' bodies mass system, the angle changes in knee and hip joints.

The most significant is the ratio indicator of positions number of the GCM of bodies system to the number of stick positions on a certain side, reflecting the tactical efficiency of an athlete.

The tactical efficiency of competitive activity, in turn, is determined by the movements coordination of the athlete's body parts in the knee and hip joints, as well as by the economy of technical actions that reflect the module of angles difference fluctuations in these joints.

For a deeper assessment of the information content of the proposed tools for competitive activity analysis in maswrestling, it is necessary to study a larger number of videos.

\section{References}

[1] Krivoruchenko E.V. Improving the technical and tactical actions of students involved in mas-wrestling. Modern High Technology. 2015, 9, pp. 141-143.

[2] Krivoshapkin P.I. Selection of exercises of special strength training in mas-wrestling. Theory and Practice of Physical Education. 2015, 9, pp. 78-82.

[3] Cherkashin I.A. Differentiated criteria for technical and tactical actions of qualified athletes in mas-wrestling using a modified simulator "lower thrust". Theory and Practice of Physical Education. 2018, 7, pp. 66-68.

[4] Arlov, A., Janković, R. and Korać, K. Motor abilities of students as predictors of their performance in the pulling rod. Facta Universitatis, Series: Physical Education and Sport. 2017, 15(1), pp. 219-225.

[5] Arus, Emeric. Biomechanics of human motion: applications in the martial arts. CRC Press. 2012. 491.

[6] Kim-Kimen A.N., Kuznetsova Z.M. The development of the traditions of ancestors to the glory of the Yakut Sports. The Russian
Journal of Physical Education and Sport. 2018, 13(2), pp. 119-123. DOI: 10.14526/02_2018_319.

[7] Alexsandr S. Kuznetsov. Russian Professor's meeting. Russian Journal of Physical Education and Sport. 2019, 14(1), pp. 17-22. DOI: DOI 10.14526/2070-4798-2019-14-1-18-24

[8] Kudrin, E.P., Duro-Daini, D.A., Yakovlev, Y.I. and Nikolaev, S.K. Evaluation criteria of offensive techniques in mas-wrestling using vertical row machine simulator. Theory and Practice of Physical Education. 2015, 10, pp.24-24.

[9] P'yanzin A.I. Kuzmin A.A., P'yanizna N.N. Formation regularities of the functional systems in case of sportsman's organism adaptation to loads. Pedagogico-psychological and medico-biological problems of physical culture and sport. 2014, vol. 1(30), pp. 130-136. DOI: 10.14526/28_2014_28. 